\title{
Superintegrable systems and Riemann-Roch theorem
}

\author{
A.V. Tsiganov \\ St. Petersburg State University, St. Petersburg, Russia \\ e-mail: andrey.tsiganov@gmail.com
}

\begin{abstract}
In algebraic geometry, there is a reduction algorithm that transforms the unreduced divisor into a unique reduced divisor, which existence is guaranteed by the Riemann-Roch theorem. We discuss application of this algorithm to construction of finite-dimensional superintegrable systems with $n$ degrees of freedom identifying coordinates of the reduced divisor with integrals of motion.
\end{abstract}

\section{Introduction}

The notion of an integrable dynamical system originates in the attempts to integrate equations of motion of the specific mechanical and astronomical problems in some explicit way. In particular, we can speak about the integrability by quadratures if we can determine all the solutions of equations of motion by means of a finite number of algebraic operations, including inversion of functions, and integrations of known functions of one variable, see [11, page 135.

For dynamical systems integrable by Abel-Jacobi quadratures these integrals have the form

$$
\int r(x, y(x)) d x
$$

where $r(x, y(x))$ is a rational function on $x$ and on algebraic function $y(x)$, which satisfies equation

$$
\Phi(x, y(x))=0
$$

with an irreducible polynomial $\Phi(x, y)$ depending on the values of integrals of motion. This integral becomes well-defined upon choosing a particular branch of solutions to (1.2) along a path of integration in the $x$-plane, that avoids the branch points where there are multiple roots. In more modern terms, one considers integral

$$
\int_{\gamma} \omega
$$

on the algebraic curve $X$ defined by equation (1.2) on the projective plane. Here $\omega$ is the restriction to $X$ of the rational differential 1-form $r(x, y) d x$, path of integration $\gamma$ depends on initial conditions and avoids the singularities of $X$ and the poles of $\omega$.

Integrals (1.1) are highly transcendental functions of the upper limit of integration and consequently are generally difficult to study directly, but in classical mechanics we usually have the sum of such integrals

$$
u(t)=\sum_{i=1}^{n} \int_{x_{0}}^{x_{i}(t)} r(x, y(x)) d x=\sum_{i=1}^{n} \int_{\gamma_{i}} \omega .
$$

The formal sum of points $P_{i}(t)=\left(x_{i}, y_{i}\right)$ on $X$ is a divisor $D(t)$ of degree $n$ moving along curve $X$.

So, when we speak of integrability by quadratures of finite-dimensional dynamical systems, we implicitly allude to replacement of motion in physical space to motion of divisors along the algebraic curves. Evolution of divisors is governed by algebraic laws such as the Bézout theorem, Abel theorem, Riemann-Roch theorem, Brill-Noether theorem, etc. It allows us to apply these algebraic laws instead of the Emmy Noether theorem for the search of additional conservation laws of dynamical system integrable by quadratures. 
For instance, in classical mechanics divisor $D(t)$ of degree $n$ on a smooth hyperelliptic curve $X$ of genus $g$ could be

- a semi-reduced divisor at $n>g$,

- a reduced divisor at $n \leq g$.

According to the Riemann-Roch theorem each semi-reduced divisor $D$ on $X$ has a unique reduced representative $D^{\prime}=\rho(D)$ of degree $g$. Movable semi-reduced divisor $D(t)$ and reduced divisor $D^{\prime}$ form the intersection divisor

$$
D(t)+D^{\prime}=0, \quad \operatorname{deg} D(t)=n, \quad \operatorname{deg} D^{\prime}=g,
$$

of $X$ with movable auxiliary curve $Y(t)$ [1]. The reduced divisor $D^{\prime}=\rho(D)$ could be

- a non trivial function $D^{\prime}(t)$ on time;

- a trivial function $D^{\prime}=$ const on time.

If $D^{\prime}$ is a constant divisor, then its coordinates are $g$ integrals of motion, which are functionally independent on $n$ integrals of motion entering into the definition of hyperelliptic curve $X$ (1.2).

In this note we want to discuss superintegrable systems having $n+g$ functionally independent integrals of motion which are associated with the Riemann-Roch theorem.

\subsection{Divisor class group}

In this section we repeat some definitions and facts from the following textbooks [5, 8 .

Let $X$ be a hyperelliptic curve of genus $g$ defined by equation

$$
X: \quad y^{2}+h(x) y=f(x),
$$

where $f(x)$ is a polynomial of degree $2 g+2$ or $2 g+1$ with distinct roots, and $h(x)$ is a polynomial with $\operatorname{deg} h \leq g$. Prime divisors are points $P_{i}=\left(x_{i}, y_{i}\right)$ on $X$ including points at infinity.

Definition 1 Divisor $D=\sum m_{i} P_{i}, m_{i} \in \mathbb{Z}$ is a formal sum of points on the curve, and degree of divisor $D$ is the sum $\sum m_{i}$ of multiplicities of points in support of the divisor.

The group of divisors $\operatorname{Div} X$ is an additive abelian group under the formal addition rule

$$
\sum m_{i} Z_{i}+\sum n_{i} Z_{i}=\sum\left(m_{i}+n_{i}\right) Z_{i}
$$

To define an equivalence relation on the divisors we use rational functions on $X$. Function $f$ is a quotient of two polynomials; each of them is zero only on a finite closed subset of codimension one in $X$, which is therefore a union of finitely many prime divisors. The difference of these two subsets defines the principal divisor div $f$ associated with function $f$. Subgroup of $\operatorname{Div} X$ consisting of the principal divisors is denoted by $\operatorname{Prin} X$.

Definition 2 Two divisors $D, D^{\prime} \in$ DivX are linearly equivalent

$$
D \approx D^{\prime}
$$

if their difference $D-D^{\prime}$ is principal divisor

$$
D-D^{\prime}=\operatorname{div}(f) \equiv 0 \quad \bmod \operatorname{Prin} X .
$$

Quotient group of $\operatorname{Div} X$ by subgroup of principal divisors $\operatorname{Prin} X$

$$
\operatorname{Pic} X=\frac{\operatorname{Div} X}{\operatorname{Prin} X}=\frac{\text { Divisors defined over } \mathrm{k}}{\text { Divisors of functions defined over } \mathrm{k}} .
$$

is called the divisor class group or the Picard group. 
Restricting to degree zero, we can also define $\operatorname{Pic}^{0} X=\operatorname{Div}^{0} X / \operatorname{Prin} X$. The groups $\operatorname{Pic} X$ and $\operatorname{Pic}^{0} X$ carry essentially the same information on $X$, since we always have

$$
\operatorname{Pic} X / \operatorname{Pic}^{0} X \cong \operatorname{Div} X / \operatorname{Div}^{0} X \cong \mathbb{Z} \text {. }
$$

The divisor class group, where the elements are equivalence classes of degree zero divisors on $X$, is isomorphic to the Jacobian of $X$. Usually divisor and its class in Pic $X$ are denoted by the same symbol D.

Some divisors on curve $X$ are more important than others, in a sense that they contain more information about the curve itself. Indeed, in order to describe equivalence classes of degree zero divisors we can use the semi-reduced and reduced divisors.

Definition 3 A semi-reduced divisor is a finite sum of prime divisors of the form

$$
D=\sum m_{i} P_{i}-D_{\infty}, \quad \operatorname{deg} D_{\infty}=\sum m_{i}
$$

where $m_{i}>0, P_{i} \neq-P_{j}$ for $i \neq j$, no $P_{i}$ satisfying $P_{i}=-P_{i}$ appears more than once, and $D_{\infty}$ is a linear combination of points at infinity.

As semi-reduced divisors are not unique in their equivalence class we have to introduce reduced divisors.

Definition 4 Semi-reduced divisor $D$ is called reduced if $\sum m_{i} \leq g$, i.e. if the sum of multiplicities is no more than the genus of curve $C$.

It follows from the Riemann-Roch theorem that for hyperelliptic curves, each equivalence class $D$ has a unique reduced representative of the form

$$
D^{\prime}=\rho(D)=P_{1}+\cdots+P_{m}-D_{\infty}, \quad \operatorname{deg} D_{\infty}=m
$$

It is enough to construction of superintegrable systems, a complete theory of divisors may be found in [5, 8.

Using reduced divisors $D$ instead their equivalence classes we can describe fast and efficient algorithms for calculations on hyperelliptic curves. Following [1, 9], in [14] Mumford determined polynomial representation of reduced divisor $D=(U(x), V(x))$, i.e. a representation of the group element in $\operatorname{Jac}(X)$ :

$$
U(x)=\prod\left(x-x_{i}\right)^{m_{i}}, \quad V\left(x_{i}\right)=y_{i}, \quad \operatorname{deg}(V)<\operatorname{deg}(U) \leq g, \quad v^{2}-f \equiv 0 \bmod u .
$$

Here monic polynomial $U(x)$ may have multiple roots, polynomial $V(x)$ is the interpolation polynomial through all the $P_{i}$ according to multiplicity $m_{i}$.

In 4] Cantor proposed the following algorithm for reduction of semi-reduced divisor $D, \operatorname{deg} D>g$ to reduced divisor $D^{\prime}, \operatorname{deg} D^{\prime}=g$ :

$$
\begin{array}{ll}
\text { Input } & \text { semi-reduced divisors } \quad D=(U, V) \quad \operatorname{deg} D>g \\
\text { Output } & \text { reduced divisor } D^{\prime}=\left(U^{\prime}, V^{\prime}\right) \quad \operatorname{deg} D^{\prime}=g
\end{array}
$$

1. while $\operatorname{deg}(U)>g$ do

$$
\begin{aligned}
& U^{\prime} \leftarrow \frac{f-h V-V^{2}}{U}, \quad V^{\prime} \leftarrow-h-V \bmod U^{\prime} \\
& U \leftarrow \operatorname{MakeMonic}\left(U^{\prime}\right), \quad V \leftarrow V^{\prime}
\end{aligned}
$$

2. $\operatorname{return}\left(U^{\prime}=U, V^{\prime}=V\right)$

This algorithm is a suitably formulated part of the more generic Abel's calculations [1] applicable to any algebraic curve. In 9] Jacobi called polynomial $U^{\prime}$ a generating function of algebraic integrals of Abel's equations.

Below we discuss a practical application of this algorithm in classical mechanics. 


\subsection{Superintegrable systems and semi-reduced divisors}

Let $X$ be a smooth projective curve of genus $g$ defined by equation

$$
X: \quad \Phi(x, y)=0
$$

over an algebraically closed field. The $n$-fold symmetric product $X(n)$ of $X$ is $n$-dimensional variety, the quotient of the $n$-fold Cartesian product $X[n]$ under action of the symmetric group on $n$ letters. The points of $X(n)$ represent naturally and one-to-one the positive divisors of degree $n$ on $X$, and so in the sequel we will let

$$
D=P_{1}+P_{2}+\ldots+P_{n}
$$

either an effective divisor or the corresponding point of $X(n)$. The higher symmetric products and the corresponding semi-reduced divisors are used to construct the Jacobian of $X$, either by excision-andglue method of Weil [25, or the projective method of Chow [13].

According to Jacobi [10] variety $X(n)$ can be considered as the $n$ dimensional Lagrangian submanifold of integrable systems. Indeed, coordinates $\left(x_{i}, y_{i}\right), i=1, . . n$ of points $P_{i}$ in the support of effective divisor $D$ satisfy $n$ so-called separation relations

$$
\Phi\left(x_{i}, y_{i}, \alpha_{1}, \cdots, \alpha_{n}\right)=0, \quad \operatorname{det}\left(\frac{\partial^{2} \Phi}{\partial x_{i} \partial \alpha_{j}}\right) \neq 0 .
$$

where $\alpha_{1}, \ldots, \alpha_{n}$ are values of $n$ integrals of motion. Solving (1.7) with respect to $\alpha_{j}$ we obtain $n$ functionally independent integrals of motion $I_{j}=\alpha_{j}$, which are in the involution with respect to the following Poisson brackets

$$
\left\{x_{i}, y_{i}\right\}=\phi_{i}\left(x_{i}, y_{i}\right)
$$

where $\phi_{i}$ are arbitrary functions, and other brackets are equal to zero [10. So, variety $X(n)$ is a Lagrangian manifold with respect to symplectic forms associated with these brackets at $\phi_{i} \neq 0$. So, Jacobi join together methods of algebraic and symplectic geometry.

Let us consider hyperelliptic curves $X$ (1.5) and semi-reduced divisor of zero degree

$$
D=P_{1}+P_{2}+\ldots+P_{n}-D_{\infty}, \quad \operatorname{deg} D_{\infty}=n,
$$

which consists of prime points $P_{i}(t)$ on $X$ with coordinates $\left(x_{i}, y_{i}\right)$. This semi-reduced divisor $D$ can be reduced to a unique reduced divisor $D^{\prime}, \operatorname{deg} D^{\prime}=g$ according to the Riemann-Roch theorem. The reduced divisor $D^{\prime}=\rho(D)$ could be a constant divisor or some non-trivial function on time.

Example 1 For instance, let us consider the well known addition formulae for the Legendrian elliptic integrals

$$
\begin{gathered}
\int \frac{d x_{1}}{\left(1+n x_{1}^{2}\right) y_{1}}+\int \frac{d x_{2}}{\left(1+n x_{2}^{2}\right) y_{2}}+\int \frac{d x_{3}}{\left(1+n x_{3}^{2}\right) y_{3}}=\ln \varphi\left(x_{1}, x_{2}, x_{3}\right)+\text { const } \\
\int \frac{\left(1-\kappa^{2} x_{1}^{2}\right) d x_{1}}{y_{1}}+\int \frac{\left(1-\kappa^{2} x_{2}^{2}\right) d x_{2}}{y_{2}}+\int \frac{\left(1-\kappa^{2} x_{3}^{2}\right) d x_{3}}{y_{3}}=\kappa^{2} x_{1} x_{2} x_{3}+\text { const } \\
\int \frac{d x_{1}}{y_{1}}+\int \frac{d x_{2}}{y_{2}}+\int \frac{d x_{3}}{y_{3}}=\text { const }
\end{gathered}
$$

where an explicit form of $\phi$ may be found in [2]. According to Abel's theorem points $P_{k}=\left(x_{k}, y_{k}\right)$ on the elliptic curve

$$
X: y^{2}=\left(1-x^{2}\right)\left(1-\kappa^{2} x^{2}\right),
$$

where

$$
x_{3}=\frac{x_{1} y_{2}+x_{2} y_{1}}{1-\kappa^{2} x_{1}^{2} x_{2}^{2}}
$$

form an intersection divisor

$$
P_{1}(t)+P_{2}(t)+P_{3}(t)=0,
$$


which consists of semi-reduce divisor $D=P_{1}(t)+P_{2}(t)$ and reduced divisor $D^{\prime}=P_{3}(t)$. Here and below for brevity we omit the points at infinity which are independent on time. The history of this geometric interpretation of Abel's results is discussed in [3, 7.

Reduced divisor $D^{\prime}$ consists of point $P_{3}$ which is a fixed point with respect to time entering into Abel's theorem for the elliptic integrals of the first kind. For elliptic integrals of second and third kinds point $P_{3}$ is a movable point.

Let us formulate the main result in this note:

Proposition 1 Let us consider integrable by quadratures dynamical system with $n$ degrees of freedom associated with the semi-reduced divisors $D(t)$ of degree $n>g$ on a hyperelliptic curve $X$ of genus $g$. If the corresponding reduced divisor $D^{\prime}$ of degree $g$ is a constant divisor, then we have superintegrable system with $n+g$ functionally independent integrals of motion.

Proof: Reduced divisor $D^{\prime}=\rho(D)$ exists according to the Riemann-Roch theorem. The coordinates of reduced divisor $D^{\prime}$ are functionally independent on $I_{j}=\alpha_{j}$ according to the Abel theorem, in which these coordinates were called "algebraic constraints". Explicit expressions for these coordinates, i.e. expressions for integrals of motion, can be obtained using standard reduction algorithm.

A set of special divisors $D$ with $\rho(D)=$ conts can be described in the framework of the BrillNoether theory of special divisors usually formulated in sheaf cohomology terms. Below we consider some examples of such special divisors and the corresponding superintegrable systems by using the Abel [1] and Jacobi [9] methods.

Example 2 In [9] Jacobi found algebraic integrals of Abel's equations associated with the basis of holomorphic differentials

$$
\begin{array}{cccc}
\frac{d x_{1}}{\sqrt{X_{1}}} & +\frac{d x_{2}}{\sqrt{X_{2}}}+ & \cdots & +\frac{d x_{n}}{\sqrt{X_{n}}}=0, \\
\frac{x_{1} d x_{1}}{\sqrt{X_{1}}} & +\frac{x_{2} d x_{2}}{\sqrt{X_{2}}}+ & \cdots & +\frac{x_{n} d x_{n}}{\sqrt{X_{n}}}=0, \\
\cdots & \cdots & \cdots & \cdots \\
\frac{x_{1}^{n-2} d x_{1}}{\sqrt{X_{1}}} & +\frac{x_{2}^{n-2} d x_{2}}{\sqrt{X_{2}}}+ & \cdots & +\frac{x_{n}^{n-2} d x_{n}}{\sqrt{X_{n}}}=0,
\end{array}
$$

where $X_{k}=X\left(x_{k}\right)$ is a polynomial of $2 n$-ts order on variable $x_{k}$.

In our terms Jacobi considere superintegrable systems with $n$ degrees of freedom associated with the semi-reduced divisor of degree $n$ on hyperelliptic curve of genus $g=n-1$ with $h(x)=0$ and $f(x)=X(x)$ in (1.5). The $n-1$ equations (1.8) define the form of trajectories of these superintegrable systems, whereas the one remaining equation of motion, for instance,

$$
\frac{x_{1}^{K} d x_{1}}{\sqrt{X_{1}}}+\frac{x_{2}^{K} d x_{2}}{\sqrt{X_{2}}}+\cdots \frac{x_{n}^{K} d x_{n}}{\sqrt{X_{n}}}=d t, \quad K>n-2=g-1,
$$

defines parametrization of the trajectories by time $t$. Of course, here we can use any differential $\omega$ on the hyperelliptic curve which is independent on holomorphic differentials $x^{j} / y d x, j<g-1$ entering into the system of Abel's equations (1.8).

At $n=2$ equation (1.8) coincides with the Euler differential equation on an elliptic curve. The corresponding superintegrable systems are discussed in [15, 17, 18, 19].

\section{Superintegrable Stäckel systems}

Let us consider Stäckel systems with $n$ degrees of freedom defined by separated relations

$$
\Phi\left(u_{j}, p_{u_{j}}\right)=p_{u_{j}}^{2}-f\left(u_{j}, \alpha_{1}, \ldots, \alpha_{n}\right)=0,
$$


where $f$ is a function on $u$ and $\alpha_{k}$, so that Stäckel matrix with entries

$$
S_{i j}=\frac{\partial \Phi\left(u_{j}, p_{u_{j}}\right)}{\partial \alpha_{i}}, \quad i, j=1, \ldots, n,
$$

is a non-degenerate matrix. Solutions of (2.9) with respect to $\alpha_{j}$ are commuting polynomials $I_{j}$ of second order on momenta $p_{u_{i}}$.

According to Stäckel [16, Hamiltonian $H=I_{1}$ and Poisson brackets $\left\{u_{i}, p_{u_{i}}\right\}=1$ generate equations of motion, which can be explicitly integrated by quadratures

$$
\sum_{i=1}^{n} \int \frac{\partial \Phi\left(u_{j}, p_{u_{j}}\right)}{\partial \alpha_{1}} \frac{d u_{j}}{p_{u_{j}}}=t
$$

and

$$
\sum_{i=1}^{n} \int \frac{\partial \Phi\left(u_{j}, p_{u_{j}}\right)}{\partial \alpha_{k}} \frac{d x_{j}}{p_{u_{j}}}=\text { const }, \quad k=2, \cdots, n .
$$

The first quadrature determines parameterization of trajectories by time $t$, other quadratures determine the form of trajectories. According to Euler [6], we can use these $n-1$ equations in order to describe systems with algebraic trajectories of motion.

Proposition 2 Equations (2.11) describe motion of the divisor $D=\sum P_{i}(t)$ of degree $n$ on a hyperelliptic curve $X$ (2.9) of degree $g$. If $n>g$ and and this semi-reduced divisor $D$ is reduced to a constant divisor $\rho(D)$, then we have superintegrable Stäckel system with $n+g$ algebraic integrals of motion.

According to Jacobi [9] the reduced divisor is a constant when quadratures (2.11) involve a complete set of holomorphic differentials on $X$, similar to (1.8). Below we consider only such constant reduced divisors.

\subsection{Maximally superintegrable systems}

Let us consider a case of $g=n-1$ and hyperelliptic curves of the form

$$
\begin{aligned}
X: \quad y^{2} & =\prod_{i=1}^{M}\left(x-e_{i}\right)\left(\sum_{j=g}^{2 g+2-M} a_{j} x^{j}+\alpha_{1} x^{K}+\alpha_{2} x^{n-2}+\alpha_{3} x^{n-3}+\cdots+\alpha_{n}\right) \\
& =\phi(x)\left(A(x)+\alpha_{1} x^{K}+H(x)\right),
\end{aligned}
$$

where $M=0, \ldots, g-2$ and $K=g, \cdots 2 g+2$ and $e_{i}, a_{j} \in \mathbb{R}$ are the parameters of our superintegrable system. Substituting

$$
x=u_{j} \quad \text { and } \quad y=\prod_{i=1}^{M}\left(u_{j}-e_{i}\right) p_{u_{j}}, \quad j-1, \ldots, n,
$$

where $u_{j}$ and $p_{u_{j}}$ are canonical coordinates $\left\{u_{j}, p_{u_{j}}\right\}=1$, into (2.12) and solving the resulting $n$ equations with respect to $\alpha_{1}, \ldots, \alpha_{n}$ we obtain $n$ independent polynomials $I_{j}=\alpha_{j}$ of second order in momenta $p_{u_{k}}$ commuting to each other

$$
\left\{I_{j}, I_{k}\right\}=0 .
$$

These integrable systems are maximally superintegrable Stäckel systems with Hamiltonian $I_{1}=\alpha_{1}$, because for curve $X$ (2.12) Stäckel's quadratures (2.11)

$$
\sum_{i=1}^{n} \int \frac{\partial \Phi\left(x_{j}, y_{j}\right)}{\partial \alpha_{k}} \frac{d x_{j}}{y}=\sum_{i=1}^{n} \int \frac{\partial H\left(u_{j}\right)}{\partial \alpha_{k}} \frac{d u_{j}}{p_{u_{i}}}=\sum_{i=1}^{n} \int \frac{u_{j}^{n-k} d u_{j}}{p_{u_{i}}}=\text { const }, \quad k=2, \cdots, n
$$

involve all the holomorphic differentials on $X$ and, therefore, we can find $n-1$ algebraic integrals of motion directly following to $[9]$. 
Example 3 In partial case $M=n=g+1$ we have two curves

$$
X: \quad \Phi(x, y)=y^{2}-\prod_{i=1}^{n}\left(x-e_{i}\right) \cdot\left(a x^{n}+\alpha_{1} x^{n-1}+\alpha_{2} x^{n-2}+\cdots+\alpha_{n}\right)=0
$$

and

$$
X: \quad \Phi(x, y)=y^{2}-\prod_{i=1}^{n}\left(x-e_{i}\right) \cdot\left(\alpha_{1} x^{n}+a x^{n-1}+\alpha_{2} x^{n-2}+\cdots+\alpha_{n}\right)=0,
$$

which describe the $n$-dimensional harmonic oscillator

$$
H=\frac{1}{2} \sum\left(p_{i}^{2}+a q_{i}^{2}\right)
$$

and Kepler problem

$$
H=\frac{1}{2} \sum p_{i}^{2}+\frac{a}{\sqrt{\sum q_{i}^{2}}}
$$

separable in elliptic coordinates in $\mathbb{R}^{n}$ :

$$
1+\sum_{i=1}^{n} \frac{q_{i}^{2}}{x-e_{i}}=\frac{U(x)}{\prod_{i=1}^{n} x-e_{i}}, \quad U(x)=\prod_{j=1}^{n}\left(x-u_{j}\right) .
$$

Here $U(x)$ is the first Mumford coordinate of the corresponding semi-reduced divisor $D$ on $X$, whereas parameters $e_{1}<e_{2} \cdots<e_{n}$ determine elliptic coordinate system

$$
u_{1}<e_{1}<u_{2}<e_{2}<\cdots<u_{n}<e_{n} .
$$

So, this partial case corresponds to the well-known maximally superintegrable systems.

Example 4 In partial case $M=n-1=g$ we have three curves

$$
\begin{array}{ll}
X: & \Phi(x, y)=y^{2}-\prod_{i=1}^{n-1}\left(x-e_{i}\right) \cdot\left(a x^{n+1}+b x_{n}+\alpha_{1} x^{n-1}+\alpha_{2} x^{n-2}+\cdots+\alpha_{n}\right)=0, \\
X: & \Phi(x, y)=y^{2}-\prod_{i=1}^{n-1}\left(x-e_{i}\right) \cdot\left(a x^{n+1}+\alpha_{1} x^{n}+b x^{n-1}+\alpha_{2} x^{n-2}+\cdots+\alpha_{n}\right)=0, \\
X: & \Phi(x, y)=y^{2}-\prod_{i=1}^{n-1}\left(x-e_{i}\right) \cdot\left(\alpha_{1} x^{n+1}+a x^{n}+b x^{n-1}+\alpha_{2} x^{n-2}+\cdots+\alpha_{n}\right)=0
\end{array}
$$

and three well-known superintegrable systems, separable in parabolic coordinates in $\mathbb{R}^{n}$

$$
x-2 q_{n}-\sum_{i=1}^{n-1} \frac{q_{i}^{2}}{x-e_{i}}=\frac{U(x)}{\prod_{i=1}^{n-1} x-e_{i}}, \quad U(x)=\prod_{j=1}^{n}\left(x-u_{j}\right) .
$$

Here $U(x)$ is the first Mumford coordinate of the corresponding semi-reduced divisor $D$ on $X$, whereas parameters $e_{1}<e_{2} \cdots<e_{n-1}$ determine local parabolic coordinate system

$$
u_{1}<e_{1}<u_{2}<e_{2}<\cdots<u_{n}
$$

and $a, b$ are parameters of potentials in the Hamiltonian. As above, this partial case corresponds to the well-known maximally superintegrable systems.

Example 5 Let us present an explicit formulae for additional integrals of motion at $n=3$ and $M=2$, when hyperelliptic curve $X(2.12)$ is defined by equation

$$
X: \quad y^{2}=x\left(x-a_{1}\right)\left(\alpha^{2} x^{4}+\beta x^{3}+\alpha_{1} x^{2}+\alpha_{2} x+\alpha_{3}\right) .
$$

Substituting

$$
x=u_{i}, \quad y=u_{i}\left(u_{i}-a_{1}\right) p_{u_{i}}, \quad i=1,2,3,
$$


into this equation and solving the resulting equations with respect to $\alpha_{j}$ we obtain standard Stäckel's integrals of motion

$$
\begin{aligned}
I_{1} & =\frac{u_{1}\left(u_{1}-a_{1}\right) p_{u_{1}}^{2}}{\left(u_{1}-u_{3}\right)\left(u_{1}-u_{2}\right)}+\frac{u_{2}\left(u_{2}-a_{1}\right) p_{u_{2}}^{2}}{\left(u_{3}-u_{2}\right)\left(u_{1}-u_{2}\right)}+\frac{u_{3}\left(u_{3}-a_{1}\right) p_{u_{3}}^{2}}{\left(u_{2}-u_{3}\right)\left(u_{1}-u_{3}\right)} \\
& -\left(u_{1}^{2}+u_{1} u_{2}+u_{1} u_{3}+u_{2}^{2}+u_{2} u_{3}+u_{3}^{2}\right) \alpha^{2}-\left(u_{1}+u_{2}+u_{3}\right) \beta, \\
I_{2} & =\frac{u_{1}\left(a_{1}-u_{1}\right)\left(u_{2}+u_{3}\right) p_{u_{1}}^{2}}{\left(u_{1}-u_{3}\right)\left(u_{1}-u_{2}\right)}+\frac{\left(a_{1}-u_{2}\right) u_{2}\left(u_{1}+u_{3}\right) p_{u_{2}}^{2}}{\left(u_{3}-u_{2}\right)\left(u_{1}-u_{2}\right)}+\frac{u_{3}\left(a_{1}-u_{3}\right)\left(u_{1}+u_{2}\right) p_{u_{3}}^{2}}{\left(u_{2}-u_{3}\right)\left(u_{1}-u_{3}\right)} \\
& +\left(u_{2}+u_{3}\right)\left(u_{1}+u_{3}\right)\left(u_{1}+u_{2}\right) \alpha^{2}+\left(u_{1} u_{2}+u_{1} u_{3}+u_{2} u_{3}\right) \beta, \\
I_{3} & =\frac{\left(u_{1}-a_{1}-\right) u_{1} u_{3} u_{2} p_{u_{1}}^{2}}{\left(u_{1}-u_{3}\right)\left(u_{1}-u_{2}\right)}+\frac{\left(u_{2}-a_{1}\right) u_{1} u_{3} u_{2} p_{u_{2}}^{2}}{\left(u_{3}-u_{2}\right)\left(u_{1}-u_{2}\right)}+\frac{\left(u_{3}-a_{1}\right) u_{1} u_{3} u_{2} p_{u_{3}}^{2}}{\left(u_{2}-u_{3}\right)\left(u_{1}-u_{3}\right)} \\
& -u_{3} u_{2} u_{1}\left(u_{1}+u_{2}+u_{3}\right) \alpha^{2}-u_{1} u_{2} u_{3} \beta .
\end{aligned}
$$

\section{Start of the reduction algorithm}

Input of the reduction algorithm is a semi-reduced divisor $D=(U, V)$ with coordinates

$$
U=\left(x-u_{1}\right)\left(x-u_{2}\right)\left(x-u_{3}\right)
$$

and

$$
\begin{aligned}
V & =\alpha x^{3}+b_{2} x^{2}+b_{1} x+b_{0} \\
& =\left(x-x_{1}\right)\left(x-x_{2}\right)\left(x-x_{3}\right) \alpha+\frac{\left(x-x_{2}\right)\left(x-x_{3}\right) y_{1}}{\left(x_{1}-x_{2}\right)\left(x_{1}-x_{3}\right)}+\frac{\left(x-x_{1}\right)\left(x-x_{3}\right) y_{2}}{\left(x_{2}-x_{1}\right)\left(x_{2}-x_{3}\right)}+\frac{\left(x-x_{1}\right)\left(x-x_{2}\right) y_{3}}{\left(x_{3}-x_{1}\right)\left(x_{3}-x_{2}\right)},
\end{aligned}
$$

where three coefficients $b_{k}$ are solutions of three algebraic equations

$$
y_{k}=V\left(x_{k}\right), \quad k=1, \ldots, 3 .
$$

According to [1] here we consider intersection of $X$ with a movable curve $Y(t)$ defined by equation $y=V(x)$, where polynomial $V(x)$ is defined by Lagrange interpolation at the points of the semi-reduced divisor $D$.

Substituting $y=V(x)$ into the equation $y^{2}-f(x)=0$ we obtain Abel's polynomial

$$
\begin{aligned}
\psi & =\left(f-V^{2}\right)=U U^{\prime} \\
& =\left(\beta-a_{1} \alpha^{2}-2 \alpha b_{2}\right) x^{5}+\left(\alpha_{1}-a_{1} \beta-2 \alpha b_{1}-b_{2}^{2}\right) x^{4}+\left(\alpha_{2}-\alpha_{1} a_{1}-2 \alpha b_{0}-2 b_{1} b_{2}\right) x^{3} \\
& +\left(\alpha_{3}-\alpha_{2} a_{1}-2 b_{0} b_{2}-b_{1}^{2}\right) x^{2}-\left(\alpha_{3} a_{1}+2 b_{0} b_{1}\right) x-b_{0}^{2},
\end{aligned}
$$

where $U^{\prime}$ is a polynomial from the first step of the reduction algorithm. Then we calculate polynomial

$$
U=\frac{\psi}{U}=\frac{\psi}{\left(x-u_{1}\right)\left(x-u_{2}\right)\left(x-u_{3}\right)}=\left(\beta-a_{1} \alpha^{2}-2 \alpha b_{2}\right) z^{2}+\cdots
$$

and make it monic in order to get coordinate $U$ of the reduced divisor $D^{\prime}$

$$
U=x^{2}+A_{1} x+A_{0}
$$

where

$$
\begin{aligned}
& A_{1}=x_{1}+x_{2}+x_{3}-a_{1}+\frac{I_{1}-a_{1}^{2} \alpha^{2}-2 a_{1} \alpha b_{2}-2 \alpha b_{1}-b_{2}^{2}}{\beta-a_{1} \alpha^{2}-2 \alpha b_{2}} \\
& A_{0}=x_{1}^{2}+x_{1} x_{2}+x_{3} x_{1}+x_{2}^{2}+x_{2} x_{3}+x_{3}^{2}-\frac{a_{1} I_{1}+2 \alpha b_{0}+2 b_{1} b_{2}-I_{2}-\left(x_{1}+x_{2}+x_{3}\right)\left(I_{1}-a_{1} \beta-2 \alpha b_{1}-b_{2}^{2}+I_{1}\right)}{\beta-a_{1} \alpha^{2}-2 \alpha b_{2}} .
\end{aligned}
$$

It is enough for our purpose and, therefore, we will not calculate the second coordinate $V$.

End of the reduction algorithm. 
Indeed, substituting the expressions for functions $b_{k}, I_{k}$ and coordinates $x_{i}, y_{i}(2.14)$ into $A_{1}, A_{0}$ we obtain two rational integrals of motion

$$
\left\{I_{1}, A_{1}\right\}=\left\{I_{1}, A_{0}\right\}=0
$$

which are independent on the polynomial integrals of motion $I_{1}, I_{2}$ and $I_{3}$. So, we get a maximally superintegrable Stäckel system.

If we identify $u_{j}$ in 2.14 ) with parabolic coordinates in $\mathbb{R}^{3}$

$$
x-2 q_{3}-\frac{q_{1}^{2}}{x}-\frac{q_{2}^{2}}{x-a_{1}}=\frac{\left(x-u_{1}\right)\left(x-u_{2}\right)\left(x-u_{3}\right)}{x\left(x-a_{1}\right)},
$$

then Hamiltonian $2 H=I_{1}$ is equal to

$$
H=\frac{p_{1}^{2}+p_{2}^{2}+p_{3}^{2}}{2}+\frac{\alpha^{2}}{2}\left(q_{1}^{2}+q_{2}^{2}+4 q_{3}^{2}\right)+\left(a_{1} \alpha^{2}-\beta\right) q_{3}+\text { const } .
$$

So, we obtain rational integrals of motion for oscillator separable in parabolic coordinates which is the well known maximally superintegrable system.

In a similar way we can construct other maximally superintegrable systems with $n$ degrees of freedom associated with hyperelliptic curves of genus $g$, so that $2 g+3 \geq n>g$. At $M \neq g, g-1$ in (2.12) these superintegrable systems are new.

\subsection{Non-maximally superintegrable systems}

If $g=n-2$, we can take one of the hyperelliptic curves of the form

$$
\begin{aligned}
X: \quad y^{2} & =\prod_{i=1}^{M}\left(x-e_{i}\right)\left(\sum_{j=g-1}^{2 g+2-M} a_{j} x^{j}+\alpha_{1} x^{K}+\alpha_{2} x^{L}+\alpha_{3} x^{n-3}+\cdots+\alpha_{n}\right) \\
& =\phi(x)\left(A(x)+\alpha_{1} x^{K}+\alpha_{2} X^{m}+H(x)\right) .
\end{aligned}
$$

In this case we have a semi-reduced divisor $D$ of degree $n$ on hyperelliptic curve $X$ of genus $g=n-2$. Substituting

$$
x=u_{j} \quad \text { and } \quad y=\prod_{i=1}^{M}\left(u_{j}-e_{i}\right) p_{u_{j}},
$$

where $u_{j}$ and $p_{u_{j}}$ are canonical coordinates $\left\{u_{j}, p_{u_{j}}\right\}=1$ into (2.16) and solving the resulting $n$ equations with respect to $\alpha_{1}, \ldots, \alpha_{n}$ we obtain $n$ independent polynomials $I_{j}=\alpha_{j}$ of second order in momenta $p_{u_{k}}$ commuting to each other

$$
\left\{I_{j}, I_{k}\right\}=0 \text {. }
$$

These integrable systems are superintegrable Stäckel systems with respect to Hamiltonians $I_{1}=\alpha_{1}$ or $I_{2}=\alpha_{2}$, because for curve $X$ (2.16) Stäckel's quadratures (2.11)

$$
\sum_{i=1}^{n} \int \frac{\partial \Phi\left(x_{j}, y_{j}\right)}{\partial \alpha_{k}} \frac{d x_{j}}{y}=\sum_{i=1}^{n} \int \frac{\partial H\left(u_{j}\right)}{\partial \alpha_{k}} \frac{d u_{j}}{p_{u_{i}}}=\sum_{i=1}^{n} \int \frac{u_{j}^{n-k} d u_{j}}{p_{u_{i}}}=\text { const }, \quad k=3, \cdots, n
$$

involve all the holomorphic differentials on $X$ and, therefore, we can find algebraic integrals of motion directly following to 9 .

Example 6 For completeness, we present one partial example of such systems at $n=3$, which was discussed in 23$]$.

Let us consider symmetric product $X[3]$ of the elliptic curve $X$ defined by equation

$$
X: \quad \Phi(x, y)=y^{2}-f(x)=0, \quad f(x)=\alpha x^{4}+\beta x^{3}+\alpha_{1} x^{2}+\alpha_{2} x+\alpha_{3},
$$


and identify coordinate of point in $X[3]$, i.e. affine coordinates of the corresponding effective divisor $D$ (1.6), with canonical coordinates in $T^{*} \mathbb{R}^{3}$

$$
x_{1}=u_{1}, \quad y_{1}=p_{u_{1}}, \quad x_{2}=u_{2}, \quad y_{2}=p_{u_{2}}, \quad x_{3}=u_{3}, \quad y_{3}=p_{u_{3}} .
$$

According to Jacobi it allows us to joint algebraic and symplectic methods of investigations.

Solving equations $\Phi\left(x_{i}, y_{i}\right)=0$ w.r.t $\alpha_{k}$ we obtain three independent integrals of motion

$$
\begin{aligned}
& I_{1}=\frac{p_{u_{1}}^{2}}{\left(u_{1}-u_{3}\right)\left(u_{1}-u_{2}\right)}+\frac{p_{u_{2}}^{2}}{\left(u_{2}-u_{3}\right)\left(u_{2}-u_{1}\right)}+\frac{p_{u_{3}}^{2}}{\left(u_{3}-u_{1}\right)\left(u_{3}-u_{2}\right)}-\left(u_{1}^{2}+u_{2}^{2}+u_{3}^{2}+u_{1} u_{2}+u_{1} u_{3}+u_{2} u_{3}\right) \alpha-\left(u_{1}+u_{2}+u_{3}\right) \beta \\
& I_{2}=-\frac{\left(u_{2}+u_{3}\right) p_{u_{1}}^{2}}{\left(u_{1}-u_{3}\right)\left(u_{1}-u_{2}\right)}-\frac{\left(u_{1}+u_{3}\right) p_{u_{2}}^{2}}{\left(u_{2}-u_{3}\right)\left(u_{2}-u_{1}\right)}-\frac{\left(u_{1}+u_{2}\right) p_{u_{3}}^{2}}{\left(u_{3}-u_{1}\right)\left(u_{3}-u_{2}\right)}+\left(u_{1}+u_{2}\right)\left(u_{1}+u_{3}\right)\left(u_{2}+u_{3}\right) \alpha+\left(u_{1} u_{2}+u_{1} u_{3}+u_{2} u_{3}\right) \beta \\
& I_{3}=\frac{u_{2} u_{3} p_{u_{1}}^{2}}{\left(u_{1}-u_{3}\right)\left(u_{1}-u_{2}\right)}+\frac{u_{1} u_{3} p_{u_{2}}^{2}}{\left(u_{2}-u_{3}\right)\left(u_{2}-u_{1}\right)}+\frac{u_{1} u_{2} p_{u_{3}}^{2}}{\left(u_{3}-u_{1}\right)\left(u_{3}-u_{2}\right)}-u_{1} u_{2} u_{3}\left(u_{1}+u_{2}+u_{3}\right) \alpha-u_{1} u_{2} u_{3} \beta .
\end{aligned}
$$

The corresponding Stäckel quadratures

$$
\begin{array}{r}
\int \frac{u_{1}^{2} d u_{1}}{\sqrt{f\left(u_{1}\right)}}+\int \frac{u_{2}^{2} d u_{2}}{\sqrt{f\left(u_{2}\right)}}+\int \frac{u_{3}^{2} d u_{3}}{\sqrt{f\left(u_{3}\right)}}=t, \\
\int \frac{u_{1} d u_{1}}{\sqrt{f\left(u_{1}\right)}}+\int \frac{u_{2} d u_{2}}{\sqrt{f\left(u_{2}\right)}}+\int \frac{u_{3} d u_{3}}{\sqrt{f\left(u_{3}\right)}}=\text { const }, \\
\int \frac{d u_{1}}{\sqrt{f\left(u_{1}\right)}}+\int \frac{d u_{2}}{\sqrt{f\left(u_{2}\right)}}+\int \frac{d u_{3}}{\sqrt{f\left(u_{3}\right)}}=\text { const. }
\end{array}
$$

involve a holomorphic differential on elliptic curve and, therefore, the arithmetic equation for intersection divisor

$$
\left(P_{1}(t)+P_{2}(t)+P_{3}(t)\right)+P_{4}=D(t)+D^{\prime}=0 .
$$

is a sum of movable divisor $D(t)$ of third degree and constant divisor $D^{\prime}$ of first degree.

\section{Start of the reduction algorithm}

Algorithm input consists of coordinates of the semi-reduced divisor $D(t)$ of third degree

$$
U=\left(x-x_{1}\right)\left(x-x_{2}\right)\left(x-x_{3}\right), \quad V=b_{2} x^{2}+b_{1} x+b_{0}
$$

where $b_{2}, b_{1}$ and $b_{0}$ are solution of the equations

$$
y_{1}=b_{2} x_{1}^{2}+b_{1} x_{1}+b_{0}, \quad y_{2}=b_{2} x_{2}^{2}+b_{1} x_{2}+b_{0}, \quad y_{3}=b_{2} x_{3}^{2}+b_{1} x_{3}+b_{0} .
$$

Substituting coordinates of the semi-reduced divisor $D$ into the polynomial

$$
U^{\prime}=\frac{f-V^{2}}{U}
$$

and making it monic, we obtain coordinates $U^{\prime}=\left(x-x_{4}\right)$ and $V^{\prime}=y_{4}$ of the reduced divisor $D^{\prime}$, where

$$
x_{4}=-x_{1}-x_{2}-x_{3}-\frac{a_{3}-2 b_{1} b_{2}}{a_{4}-b_{2}^{2}}, \quad y_{4}=-V\left(x_{4}\right) .
$$

\section{End of the reduction algorithm.}

After substitution (2.18) we obtain rational functions $x_{4}$ and $y_{4}$ on phase space $T^{*} \mathbb{R}^{3}$

$$
x_{4}=-u_{1}-u_{2}-u_{3}-\frac{\beta-2 b_{1} b_{2}}{\alpha-b_{2}^{2}}, \quad y_{4}=-\left(b_{2} x_{4}^{2}+b_{1} x_{4}+b_{0}\right),
$$


where

$$
\begin{aligned}
& b_{2}=\frac{\left(u_{2}-u_{3}\right) p_{u_{1}}+\left(u_{3}-u_{1}\right) p_{u_{2}}+\left(u_{1}-u_{2}\right) p_{u_{3}}}{\left(u_{1}-u_{2}\right)\left(u_{1}-u_{2}\right)\left(u_{2}-u_{3}\right)}, \\
& b_{1}=-\frac{\left(u_{2}^{2}-u_{3}^{2}\right) p_{u_{1}}-\left(u_{3}^{2}-u_{1}^{2}\right) p_{u_{2}}-\left(u_{1}^{2}-u_{2}^{2}\right) p_{u_{3}}}{\left(u_{1}-u_{2}\right)\left(u_{1}-u_{2}\right)\left(u_{2}-u_{3}\right)}, \\
& b_{0}=\frac{u_{2} u_{3}\left(u_{2}-u_{3}\right) p_{u_{1}}+u_{1} u_{3}\left(u_{3}-u_{1}\right) p_{u_{2}}+u_{1} u_{2}\left(u_{1}-u_{2}\right) p_{u_{3}}}{\left(u_{1}-u_{2}\right)\left(u_{1}-u_{2}\right)\left(u_{2}-u_{3}\right)} .
\end{aligned}
$$

These functions are the first integrals of the dynamical system determined by Hamiltonians $I_{1}$ or $I_{2}$ associated with nonholomorphic differentials on $X$

$$
\left\{I_{1}, x_{4}\right\}=\left\{I_{1}, y_{4}\right\}=0 \quad \text { and } \quad\left\{I_{2}, x_{4}\right\}=\left\{I_{2}, y_{4}\right\}=0 .
$$

Complete algebra of the first integrals is discusses in [23].

\section{Effective divisors with multiple points}

In Section 2 we consider the $n$-fold symmetric product $X(n)$ of hyperelliptic curve $X$ and effective divisors $D$ (1.6) of the form

$$
D=P_{1}+P_{2}+\ldots+P_{n} .
$$

If we take generic positive divisors

$$
D=m_{1} P_{1}+m_{2} P_{2}+\cdots+m_{n} P_{n}, \quad m_{i} \in \mathbb{Z}_{+}
$$

associated with sums of integrals of the holomorphic differentials on $X$

$$
m_{1} \int \frac{x_{1}^{k} d x_{1}}{y_{1}}+m_{2} \int \frac{x_{2}^{k} d x_{2}}{y_{2}}+\cdots+m_{n} \int \frac{x_{n}^{k} d x_{2}}{y_{n}}=\int \frac{x_{1}^{k} d x_{1}}{y_{1} / m_{1}}+\int \frac{x_{2}^{k} d x_{2}}{y_{2} / m_{2}}+\cdots+\int \frac{x_{n}^{k} d x_{2}}{y_{n} / m_{n}},
$$

we can "restore" the symmetry between points by using substitution

$$
\frac{y_{i}}{m_{i}} \rightarrow y_{i}, \quad i=1, \ldots, n,
$$

that corresponds to a noncanonical transformation of variables in phase space of the Stäckel integrable systems (2.13)

$$
p_{u_{i}} \rightarrow \frac{p_{u_{i}}}{m_{i}}, \quad i=1, \ldots, n .
$$

So, all the superintegrable systems described in Section 2 remain superintegrable after the noncanonical transformations (3.22), see discussion in [20, 22, 23].

Example 7 Let us take 3D oscillator from Example 5 and make the noncanonical transformation (3.22) of the corresponding momenta

$$
p_{u_{1}} \rightarrow \frac{p_{u_{1}}}{2}, \quad p_{u_{2}} \rightarrow p_{u_{2}}, \quad p_{u_{3}} \rightarrow p_{u_{3}} .
$$

This transformation changes metric in Hamiltonian (2.15)

$$
\begin{aligned}
I_{1} & =\frac{u_{1}\left(u_{1}-a_{1}\right)}{\left(u_{1}-u_{3}\right)\left(u_{1}-u_{2}\right)} \frac{p_{u_{1}}^{2}}{4}+\frac{u_{2}\left(u_{2}-a_{1}\right)}{\left(u_{3}-u_{2}\right)\left(u_{1}-u_{2}\right)} p_{u_{2}}^{2}+\frac{u_{3}\left(u_{3}-a_{1}\right)}{\left(u_{2}-u_{3}\right)\left(u_{1}-u_{3}\right)} p_{u_{3}}^{2} \\
& -\left(u_{1}^{2}+u_{1} u_{2}+u_{1} u_{3}+u_{2}^{2}+u_{2} u_{3}+u_{3}^{2}\right) \alpha^{2}-\left(u_{1}+u_{2}+u_{3}\right) \beta,
\end{aligned}
$$

and doubles the first point in the movable effective divisor

$$
D=2 P_{1}(t)+P_{2}(t)+P_{3}(t) .
$$




\section{Start of the reduction alghorithm}

Input of the reduction algorithm is a semi-reduced divisor $D=(U, V)$ with coordinates

$$
U=\left(x-u_{1}\right)^{2}\left(x-u_{2}\right)\left(x-u_{3}\right)
$$

and

$$
V=b_{3} x^{3}+b_{2} x^{2}+b_{1} x+b_{0}
$$

where four coefficients $b_{k}$ are solutions of the four algebraic equations

$$
y_{k}=V\left(x_{k}\right), \quad k=1 . .3, \quad \text { and } \quad \frac{d V}{d x}\left(x_{1}\right)=\frac{1}{2 y_{1}} \frac{d f}{d x}\left(x_{1}\right) .
$$

due to the standard Hermite interpolation procedure. In order to get the first coordinate of the reduced divisor $D^{\prime}$ we have to calculate polynomial

$$
U^{\prime}=\frac{f-V^{2}}{U}=\left(\alpha^{2}-b_{3}^{2}\right)\left(x^{2}+A_{1} x+A_{0}\right)
$$

and make it monic

$$
U=x^{2}+A_{1} x+A_{0} .
$$

Coefficients of this polynomial are equal to

$$
A_{1}=2 x_{1}+x_{2}+x_{3}-\frac{\left(a_{1} \alpha^{2}+2 b_{2} b_{3}-b e t a\right.}{\alpha^{2}-b_{3}^{2}}
$$

and

$$
\begin{aligned}
A_{0} & =\frac{2 x_{1}^{2}+x_{2}^{2}+x_{3}^{2}+A_{1}^{2}-a_{1}^{2}}{2}+\frac{I_{1}}{\alpha^{2}-b_{3}^{2}} \\
& -\frac{\alpha^{2}\left(2 a_{1}^{2} b_{3}^{2}+4\left(a_{1} b_{2}+b_{1}\right) b_{3}+2 b_{2}^{2}\right)+\beta^{2}-2 b_{3}\left(a_{1} b_{3}+2 b_{2}\right) \beta-b_{3}^{2}\left(a_{1}^{2} b_{3}^{2}+4 b_{1} b_{3}-2 b_{2}^{2}\right)}{\left(\alpha^{2}-b_{3}^{2}\right)^{2}}
\end{aligned}
$$

It is enough for our purpose and, therefore, we omit for brevity calculation of the second coordinate of $D^{\prime}$

\section{End of the reduction algorithm.}

Substituting expressions for functions $b_{k}, I_{k}$ and coordinates $x_{i}, y_{i}$ (2.14) into $A_{1}, A_{0}$ we obtain two rational integrals of motion

$$
\left\{I_{1}, A_{1}\right\}=\left\{I_{1}, A_{0}\right\}=0,
$$

which are independent of the Stäckel integrals of motion $I_{1}, I_{2}$ and $I_{3}$. Complete algebra of polynomial $I_{1}, I_{2}, I_{3}$ and rational $A_{0}, A_{1}$ integrals of motion are the polynomial algebra similar to the algebras from 23 .

Example 8 In order to present calculations with a few rounds in a do - loop, we consider a 2D oscillator separable in parabolic coordinates on the plane

$$
u_{1}=q_{2}-\sqrt{q_{1}^{2}+q_{2}^{2}}, \quad u_{2}=q_{2}+\sqrt{q_{1}^{2}+q_{2}^{2}}, \quad u_{1}<0<u_{2} .
$$

Noncanonical transformation (3.22) of the corresponding momenta

$$
p_{u_{1}}=\frac{p_{2}}{2}-\frac{q_{2}+\sqrt{q_{1}^{2}+q_{2}^{2}}}{2 q_{1}} p_{1}, \quad p_{u_{2}}=\frac{p_{2}}{2}-\frac{q_{2}-\sqrt{q_{1}^{2}+q_{2}^{2}}}{2 q_{1}} p_{1}
$$

transforms original Hamiltonian

$$
H=2 I_{1}=\frac{p_{1}^{2}+p_{2}^{2}}{2}-2 \alpha^{2}\left(q_{1}^{2}+4 q_{2}^{2}\right)
$$


to the Hamiltonian

$$
H_{m_{1} m_{2}}=\frac{\left(m_{1}^{2}+m_{2}^{2}\right)\left(p_{1}^{2}+p_{2}^{2}\right)}{4 m_{1}^{2} m_{2}^{2}}-\frac{\left(m_{1}^{2}-m_{2}^{2}\right)\left(q_{2} p_{1}^{2}-2 q_{1} p_{1} p_{2}-q_{2} p_{2}^{2}\right)}{4 m_{1}^{2} m_{2}^{2} \sqrt{q_{1}^{2}+q_{2}^{2}}}-2 \alpha^{2}\left(q_{1}^{2}+4 q_{2}^{2}\right),
$$

associated with an effective divisor $D=m_{1} P_{1}+m_{2} P_{2}$ on elliptic curve

$$
E: \quad \Phi(x, y)=y^{2}-f(x)=0, \quad f(x)=x\left(\alpha^{2} x^{3}+I_{1} x+I_{2}\right)
$$

It follows from the Riemann-Roch theorem that Hamiltonian $H_{m}$ remains superintegrable, i.e. it is in involution with two independent integrals of motion. The first of them is given by $I_{2}$, the second independent integral of motion is given by coordinates of the constant reduced divisor $P_{3}$ in the equation

$$
m_{1} P_{1}(t)+m_{2} P_{2}(t)+P_{3}=0 .
$$

Below we show the reduction algorithm at $m_{1}=3$ and $m_{2}=1$.

\section{Start of the reduction alghorithm}

Input of the reduction algorithm is the following coordinates of the semi-reduced divisor $D$ of fourth degree:

$$
U(x)=\left(x-x_{1}\right)^{3}\left(x-x_{1}\right), \quad V(x)=b_{3} x^{3}+b_{2} x^{2}+b_{1} x+b_{0} .
$$

Because polynomial $V(x)$ is a Hermite interpolation polynomial, its four coefficients $b_{k}$ are solutions of the four equations

$$
y_{1}=V\left(x_{1}\right), \quad \frac{d V}{d x}\left(x_{1}\right)=\frac{1}{2 y_{1}} \frac{d f}{d x}\left(x_{1}\right), \quad \frac{d^{2} V}{d x^{2}}\left(x_{1}\right)=\frac{1}{2 y_{1}} \frac{d^{2} f}{d x^{2}}-\frac{1}{4 y_{1}^{3}}\left(\frac{d f}{d x}\left(x_{1}\right)\right)^{2} . \quad y_{2}=V\left(x_{2}\right)
$$

We start the first round of the do-loop with calculation of coordinates of the semi-reduced divisor of second degree. We calculate

$$
\begin{aligned}
U^{\prime} & =\frac{f-V^{2}}{U}=-b_{3}^{2} x^{2}-b_{3}\left(3 b_{3} x_{1}+b_{3} x_{2}+2 b_{2}\right) x \\
& -b_{3}^{2}\left(6 x_{1}^{2}+3 x_{1} x_{2}+x_{2}^{2}\right)+-b_{3}\left(6 b_{2} x_{1}+-2 b_{2} x_{2}+2 b+1\right)+\alpha^{2}-b_{2}^{2}
\end{aligned}
$$

and make it monic

$$
U=\frac{U^{\prime}}{-b_{3}^{2}}=x^{2}+A_{1} x+A_{0} .
$$

Then we have to calculate second coordinate of the semi-reduced divisor of second degree

$$
V^{\prime}=-V \bmod U^{\prime}=-\left(b_{3} x^{3}+b_{2} x^{2}+b_{1} x+b_{0} \bmod \left(-b_{3}^{2} x^{2}-b_{3}^{2} x A_{1}-b_{3}^{2} A_{2}\right) .\right.
$$

Using standard modular arithmetic with polynomials we obtain

$$
V^{\prime}=\alpha^{2} x^{2}+B_{1} x+B_{0}
$$

where

$$
B_{1}=-b_{3} A_{1}^{2}+\left(\alpha+b_{2}\right) A_{1}+b_{3} A_{0}-b_{1}, \quad B_{1}=\left(\alpha+b_{2}-b_{3} A_{1}\right) A_{0}-b_{0},
$$

and put $V=V^{\prime}$.

At the second round of the do-loop, we use coordinates of semi-reduced divisor of second degree

$$
U=x^{2}+A_{1} x+A_{0}, \quad V=\alpha^{2} x^{2}+B_{1} x+B_{0}
$$

in order to obtain polynomial

$$
U^{\prime}=\frac{f-V^{2}}{U}=-2 \alpha B_{1}(z-K)
$$


and make it monic

$$
U=z-x_{3}, \quad x_{3}=\frac{\left.\alpha\left(2 B_{0}-2 A_{1} B_{1}\right)+B_{1}^{2}-I_{1}\right)}{2 \alpha B_{1}} .
$$

As a result, we get abscissa $x_{3}$ of the reduced divisor $D^{\prime}=P_{3}$ of degree $g=1$ that is enough for our purpose.

\section{End of the reduction algorithm.}

Using expressions for $A_{1,0}(3.25), B_{1,0}(3.26)$ and $b_{k}(3.24)$ we obtain abscissa $x_{3}$ (3.27) as a rational function of coordinates of points $x_{1,2}$ and $y_{1,2}$ on curve $E$. Then, substituting

$$
x_{1}=u 1, \quad y_{1}=u_{1} \frac{p_{u_{1}}}{3} \quad x_{2}=u_{2}, \quad y_{2}=u_{2} p_{u_{2}},
$$

we get a bulky rational function on phase space $T^{*} \mathbb{R}^{2}$, which we do not present here for brevity.

It is easy to verify that this function commutes with Hamiltonian $H_{31}$

$$
2 H_{31}=I_{1}=\frac{u_{1} p_{u_{1}}^{2}}{9\left(u_{1}-u_{2}\right)}+\frac{u_{2} p_{u_{2}}^{2}}{u_{2}-u_{1}}-\alpha^{2}\left(u_{1}^{2}+u_{1} u_{2}+u_{2}^{2}\right), \quad\left\{I_{1}, x_{3}\right\}=0,
$$

and it is functionally independent of the second Stäckel integral of motion

$$
I_{2}=\frac{u_{1} u_{2} p_{u_{1}}^{2}}{9\left(u_{2}-u_{1}\right)}+\frac{u_{1} u_{2} p_{u_{2}}^{2}}{u_{1}-u_{2}}+\alpha^{2} u_{1} u_{2}\left(u_{1}+u_{2}\right), \quad\left\{I_{2}, x_{3}\right\} \neq 0
$$

using a modern computer algebra system.

\section{Conclusion}

Integrability by quadratures is related to integration of function of the one variable. For algebraic integrals this variable can be identified with a point on the corresponding algebraic curve, that allows us to study the evolution of points along curves instead of motion in phase space. Algebraic laws determine the constants of this evolution, which can be identified with integrals of motion in phase space.

In our previous papers [17, 18, 19, 20, 21, 22, 23, 24] we present some superintegrable systems with two degrees of freedom. In this note we discuss superintegrable systems with $n$-degrees of freedom for which additional integrals of motion exist due to the Riemann-Roch theorem. Our main aim is to show that such first integrals may be easily calculated using a standard reduction algorithm.

We believe that the combination of this approach based on the reduction algorithm and approach based on the bi-Hamiltonian geometry [12] allow us to obtain additional integrals of motion without tedious algorithmic calculations.

The work was supported by the Russian Science Foundation (project 18-11-00032).

\section{References}

[1] Abel N. H., Mémoire sure une propriété générale d'une classe très éntendue de fonctions transcendantes, Oeuvres complétes, Tome I, Grondahl Son, Christiania, 1881, pages 145-211, available from http://archive.org/details/OEuvresCompletesDeNielsHenrikAbel1881_12/page/n167.

[2] Barnum H.H., Abel's theorem and the addition formulae for elliptic integrals, Annals of Mathematics, Second Series, v. 11, n. 3, pp. 103-114, 1910, available from https://www.jstor.org/stable/1967324.

[3] Bliss G.A., Algebraic functions and their divisors, Annals of Mathematics, Second Series, v. 26, n. 1/2, pp. 95-124, 1924, available from https://www.jstor.org/stable/1967747.

[4] Cantor D.G., Computing in the Jacobian of a hyperelliptic curve, Mathematics of computation, v.48, n.177, pp.95-101, 1987. 
[5] Eisenbud D., Harris J., 3264 and all that: A second course in algebraic geometry, pp. 632. Cambridge University Press, 2016.

[6] Euler L., Probleme un corps étant attiré en raison réciproque quarrée des distances vers deux points fixes donnés, trouver les cas oú la courbe décrite par ce corps sera algébrique, Mémoires de l'academie des sciences de Berlin v.16, pp. 228-249, 1767, available from http://eulerarchive.maa.org/docs/originals/E337.pdf.

[7] Griffiths P., The Legacy of Abel in Algebraic Geometry, in The Legacy of Niels Henrik Abel, Ed. Laudal and Piene, pp. 179-205, Springer, Berlin-Heidelberg, 2004.

[8] Hartshorne R., Algebraic geometry, volume 52 of Graduate Texts in Mathematics, Springer-Verlag, New York, 1977.

[9] Jacobi C. G. J., Über eine neue Methode zur Integration der hyperelliptischen Differentialgleichungen und über die rationale Formihrer vollständigen algebraischen Integralgleichungen, J. Reine Angew. Math., v.32, pp.220-227, 1846, available from https://gallica.bnf.fr/ark:/12148/bpt6k90215d/f147.image

[10] Jacobi C.G.J., Vorlesungen über dynamik, Berlin, G.Reimer, 1884.

[11] Kozlov, V.V., Dynamical Systems X: General Theory of Vortices, Encyclopaedia Math. Sci., v. 67, Berlin: Springer, 2003.

[12] Maciejewski A.J., Przybylska M., Tsiganov A.V., On algebraic construction of certain integrable and super-integrable systems, Physica D, v. 240, p.1426-1448, 2011.

[13] Mattuck A., Symmetric products and Jacobians, American Journal of Mathematics, v. 83, no. 1, pp. 189-206, 1961, available from https://www.jstor.org/stable/2372727.

[14] Mumford D., Tata Lectures on Theta II, Birkhäuser, 1984.

[15] Onofri, E. and Pauri, M., Search for periodic hamiltonian flows: A generalized Bertrands theorem, J. Math. Phys., v. 19( 9), pp. 1850-1858, 1978.

[16] Stäckel P., Über die Integration der Hamilton-Jacobischen Differential Gleichung Mittelst Separation der Variabeln, Habilitationsschrift, Halle, 26pp., 1891, available from http://archiv.ub.uni-heidelberg.de/volltextserver/12758/1/staeckel_habil.pdf.

[17] Tsiganov A.V., On maximally superintegrable systems, Reg. Chaot. Dyn., v.13, pp.178-190, 2008.

[18] Tsiganov A.V., Addition theorems and the Drach superintegrable systems, J. Phys. A: Math. Theor., v. 41, 335204, 2008.

[19] Tsiganov A.V., Leonard Euler: addition theorems and superintegrable systems, Reg. Chaot. Dyn., v.14(3), pp.389-406, 2009.

[20] Tsiganov A.V., Transformation of the Stäckel matrices preserving superintegrability, Journal of Mathematical Physics, v.60, 042701, 2019.

[21] Tsiganov A.V., Superintegrable systems with algebraic and rational integrals of motion, Theor. Math. Phys., v.199, n.2, pp. 659-674, 2019.

[22] Tsiganov A.V., Elliptic curve arithmetic and superintegrable systems, Physica Scripta, v.94, 085207, 2019.

[23] Tsiganov A.V., The Kepler problem: polynomial algebra of non-polynomial first integrals, Regular and Chaotic Dynamics, v.24, pp.353-369, 2019.

[24] Tsiganov A.V., Discretization and superintegrability all rolled into one, arXiv:1902.03884, 2019.

[25] Weil A., Variét és ab éliennes et courbes alg ébriques, Actualités Sci. Ind., no. 1064, Publ. Inst. Math. Univ. Strasbourg, 1946 and Hermann et Cie., Paris, 1948. 